\title{
Effect of cyclooxygenase-2 inhibition on human Helicobacter pylori gastritis: mechanisms underlying gastrointestinal safety and implications for cancer chemoprevention
}

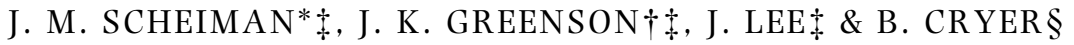 \\ *Division of Gastroenterology, Department of Internal Medicine, $†$ Department of Pathology, and the $\ddagger$ Comprehensive Cancer \\ Center, University of Michigan, Ann Arbor, Michigan, USA; §Division of Gastroenterology, Department of Internal \\ Medicine, University of Texas South-western, Dallas Veteran Affairs Medical Center, Dallas, Texas, USA
}

Accepted for publication 22 January 2003

\section{SUMMARY}

Cyclooxygenase (COX)-2 expression and prostaglandin production is increased by Helicobacter pylori infection. Non-selective COX inhibitors reduce prostaglandins and mucosal proliferation in infected mucosa and may reduce gastric cancer risk, but ulceration precludes their use. COX-2 inhibitors cause fewer ulcers and may be chemopreventive. Physiological studies of COX-2 inhibitors in humans with $H$. pylori infection have not been performed.

Aim: To study the impact of COX-2 specific inhibition on gastric prostaglandin levels, H. pylori gastritis and proliferation.

Methods: Twenty infected (eight males, 12 females; age $38 \pm 1.8$ ) and six uninfected (four males, two females; age $36 \pm 3.5$ ) healthy volunteers received rofecoxib $25 \mathrm{mg}$ daily for 14 days. Endoscopic biopsies were evaluated for prostaglandin $\mathrm{E}_{2}\left(\mathrm{PGE}_{2}\right)$ content, gastritis and proliferation.
Results: Before drug therapy, compared to uninfected, H. pylori-infected subjects had significantly higher: (a) gastric mucosal $\mathrm{PGE}_{2}(\mathrm{pg} / \mathrm{mg}$ tissue) in the gastric body and antrum, (b) H. pylori score in body and antrum and (c) mid-gland proliferation index in antrum and body. The COX-2 inhibitor did not significantly affect $\mathrm{PGE}_{2}$ levels, gastritis scores or proliferation indices in the body or antrum in the $H$. pylori-positive or -negative subjects.

Conclusion: The predominant source of increased gastric $\mathrm{PGE}_{2}$ in $H$. pylori infection appears to be COX-1-derived. In non-ulcerated $H$. pylori gastritis, $\mathrm{COX}-2$ inhibition does not affect cellular proliferation. Rofecoxib's lack of effect on gastric prostaglandin levels and proliferation in H. pylori-infected mucosa may explain the absence of an increased ulcer risk among COX-2 inhibitor users with $H$. pylori infection. The lack of significant effect on intermediate biomarkers raises uncertainty regarding the potential of specific COX-2 inhibitors for chemoprevention of gastric cancer.

\section{INTRODUCTION}

Infection with Helicobacter pylori causes characteristic mucosal inflammation and is associated with diverse clinical outcomes, including peptic ulceration and

Correspondence to: Dr J. M. Scheiman, University of Michigan Medical Center, 3912 Taubman Center Box 0362, Ann Arbor, Michigan 481090362, USA.

E-mail: jscheima@umich.edu increased risk for malignancy. Recognition of the increased cancer risk associated with the infection has led to the organism's classification by the World Health Organization as a class I carcinogen. ${ }^{1}$ There are ongoing clinical trials to determine if eradication of the organism can halt progression from this premalignant condition to neoplastic transformation. ${ }^{2,3}$ While the evidence that eradication provides an effective cancer risk reduction remains to be established, 
reinfection after eradication is common in those parts of the world where $\mathrm{H}$. pylori gastritis and associated cancer risk is greatest. This consideration raises concern to develop chemopreventive strategies to reduce $H$. pylori associated cancer risks other than antibiotic therapy.

The use of non-steroidal anti-inflammatory drugs (NSAIDs) has been identified by epidemiological studies as a possible protective factor for the development of gastrointestinal neoplasms, particularly colorectal cancer, but also gastric and oesophageal cancer. ${ }^{4}$ The putative mechanisms of this effect have been explored, and the induction of the cyclooxygenase (COX)-2 isoform has been implicated in cancer risk, particularly for colonic and oesophageal carcinogenesis. Both non-selective NSAIDs and COX-2-specific inhibitors have demonstrated reduction in colorectal polyp formation, suggesting a potential chemopreventive role for these agents.

In the case of gastric cancer, up-regulation of $\mathrm{COX}-2$ has been observed in both $\mathrm{H}$. pylori gastritis and gastric epithelial neoplasms. ${ }^{5,6}$ While NSAIDs have been demonstrated to reduce $H$. pylori-associated enhanced epithelial prostaglandin production and associated hyperproliferation, ${ }^{7,8}$ the increased risk of ulcer formation associated with these medications precludes their rational use as a chemopreventive agent.

Alternatively, COX-2-specific inhibitors appear to cause minimal endoscopic upper gastrointestinal injury, similar to placebo, and have a significantly reduced rate of clinically significant complicated gastrointestinal events compared to traditional NSAIDs. ${ }^{9,}{ }^{10}$ Rofecoxib does not effect normal human gastric mucosal prostaglandin production, confirming that COX-1-derived products predominate in the non- $H$. pylori-infected stomach. ${ }^{11}$ Similarly, although the relation between NSAIDs and H. pylori infection with regard to ulcer risk remains controversial, there is good evidence that H. pylori infection does not increase ulcer risk among users of COX-2-specific inhibitors. ${ }^{12}$ Although COX-2 is induced in the human gastric epithelium in H. pylori infection, the function and contribution of prostaglandins derived from this isoform in this setting has not been assessed. ${ }^{13}$

To evaluate the impact of specific COX-2 inhibition in the setting of the H. pylori gastric inflammation, we studied the effect of rofecoxib on mucosal prostaglandins, gastric epithelial cellular proliferation and measures of gastric inflammation in asymptomatic healthy H. pylori-infected and non-infected human volunteers. Our results suggest that despite evidence for up-regulation of COX-2, COX-1-derived prostaglandins are the predominant source of increased prostaglandin levels in H. pylori gastritis. These data support the safety of COX-2-specific inhibitors in the setting of $H$. pylori infection, but suggest that dual COX inhibition may be required to reduce gastric epithelial proliferation when COX inhibitors are used for their chemopreventive effects.

\section{METHODS}

Design

This was a prospective, single blind study evaluating mucosal prostaglandin levels, histology and proliferation before and after treatment with rofecoxib $25 \mathrm{mg}$ daily in healthy volunteers. The pathologist and all laboratory personnel were unaware of treatment allocation by the coding of specimens by the study coordinator. The Institutional Review Boards for Human Studies at the University of Michigan and the Dallas VA Medical Center approved the study.

\section{Subjects}

We recruited 26 (20 with H. pylori infection) healthy volunteers of either sex. Patients had to be free of chronic medication use and past history of gastrointestinal disease. Also excluded from participation were those who had taken an NSAID, aspirin or antisecretory agent within 14 days of study, pregnant subjects, those previously treated for the eradication of H. pylori and those allergic to NSAIDs or rofecoxib. H. pylori infection was assessed by serology and confirmed by rapid urease testing (RUT) of gastric biopsies at the time of endoscopy (described below). All women of childbearing potential underwent serum pregnancy testing prior to enrolment.

\section{Endoscopy}

After an overnight fast, subjects' throats were treated with topical anaesthetic (pontocaine) and sedation was provided by intravenous midazolam. Once the subject was sedated an endoscope was introduced through the mouth and passed into the oesophagus, stomach and duodenum, where gastric and duodenal mucosal injury was evaluated for the size and number of erosions and ulcers. An ulcer was defined as a mucosal break $\geq 3 \mathrm{~mm}$ with unequivocal depth. An erosion was defined as a 
mucosal break $<3 \mathrm{~mm}$. Any subject with an ulcer or erosions at baseline endoscopy was excluded from further study.

Following endoscopic assessment of mucosal injury, mucosal biopsies were obtained away from areas of injury in the gastric antrum and body for evaluation for histology, proliferative markers and for measurement of gastric prostaglandin $\mathrm{E}_{2}\left(\mathrm{PGE}_{2}\right)$ content, as previously described. ${ }^{14}$ An additional antral mucosal biopsy was obtained for RUT (CLOtest, Tri-Medical Specialists, Lenexa, KS).

\section{Treatment and follow-up endoscopy}

On the day following baseline endoscopy and biopsy, subjects received rofecoxib (Merck and Co, Inc., Whitehouse Station, NJ) $25 \mathrm{mg}$ daily for 14 days. On the morning of the fourteenth day of rofecoxib, 2-3 h after the final dose, endoscopy with assessment of mucosal injury and biopsy of gastric body and antral mucosa was repeated in a manner similar to the baseline endoscopy. Care was taken to avoid sampling tissue near an endoscopic abnormality.

\section{Biopsy processing}

Gastric mucosal prostaglandin assay. Tissue extraction and measurements of prostaglandins were performed separately on antral and body samples by radioimmunoassay according to a previously described method. ${ }^{14}$ Briefly, frozen biopsy specimens were crushed, homogenized in ethanol and then centrifuged. The resultant supernatant was then acidified with acetic acid. Prostaglandin fractions were then extracted by column elution through a reverse-phase octadecylsilane-bonded silica gel and reconstituted in a phosphated saline buffer. Radioimmunoassay was performed by incubating the reconstituted tissue prostaglandins with the corresponding ${ }^{3} \mathrm{H}$-prostaglandin and the corresponding anti-sera. After incubation, bound counts were determined by liquid scintillation spectrometry. Standard curves were constructed using known amounts of $\mathrm{PGE}_{2}$. Our investigations have previously reported intra-assay variations of $6.5 \%$ and inter-assay variations of $12.4 \%$ for radioimmunoassays of $\mathrm{PGE}_{2}$, respectively. ${ }^{14}$

Pathology. At each endoscopy, two biopsies from both the antrum and body were placed in separately labelled containers of formalin. Tissues were fixed for $48-72 \mathrm{~h}$ and then embedded in paraffin. A single haematoxylin and eosin stained section was made from each block and was evaluated blindly by a single pathologist for the presence of acute and chronic inflammation, atrophy, intestinal metaplasia and $H$. pylori organisms according to the updated Sydney System. ${ }^{15}$ Severity of gastritis was scored on a 0-3-point scale using the 5-dimensional Sydney classification system.

Immunohistochemistry. Mucosal proliferation was assessed by staining 5 micron tissue sections for Ki67, a cell proliferation associated antigen (monoclonal antibody M7240 at 1:100, Dako Corp. Carpenteria, CA) using a strepavidin biotin labelling kit (LSAB + Dako Corp. Carpenteria, CA) with diaminobenzidine as a chromagen. The tissue was pretreated with citrate buffer $\mathrm{pH} 6.0$ and microwaved for $10 \mathrm{~min}$ under pressure. The antibody was incubated for $30 \mathrm{~min}$ at room temperature. The number of positive-staining nuclei was counted in the surface, proliferative and deep zones of biopsies from both the antrum and body. Biopsies were scanned at low power to detect the areas with the most positive staining cells. Consecutive counts were then performed in each area until a minimum of 400 cells were counted at each location within the antral and body mucosae.

\section{Statistical analysis}

The primary outcome measures were $\mathrm{PGE}_{2}$ (a continuous measurement) and proliferation indices (\% stained cells). The secondary outcome measures were the severity of gastritis. Results are presented in the form: mean (s.e.) for $\mathrm{PGE}_{2}$ (based on untransformed data) and proliferation indices. Statistical analysis was done using SAS v8.2 (North Carolina). A two-tailed P-value of 0.01 or less was considered to be statistically significant, to correct for the multiplicity of testing. No further multiple comparison adjustment was made.

A natural logarithmic transformation was applied to $\mathrm{PGE}_{2}$ to achieve normality for analysis. Two sample $t$-tests were used to assess log-scale $\mathrm{PGE}_{2}$ differences between groups at baseline. Generalized linear models were fitted to the data using generalized estimating equation approach to account for both within and between subjects variations derived from repeated measurements. To compare the group effect over time, the generalized linear models included a group factor, a time factor, a measurement location factor (antrum 
vs. body) and all pairwise and three-way interactions. This sophisticated analysis allows more subtle differences among the groups to be detected. A Gaussian distribution was assumed for the log-scale $\mathrm{PGE}_{2}$. For the proliferation indices a binomial distribution was assumed for the number of stained cells. Least-square means were used for multiple comparisons when a significant interaction term was observed. Pearson or Spearman correlation coefficients were used to assess the relationship between $\mathrm{PGE}_{2}$, proliferation indices and gastritis severity where appropriate. Wilcoxon ranksum test and Wilcoxon sign-rank test were used to assess the gastritis severity differences between groups and within groups, respectively. Furthermore, the Mantel-Haenszel statistics was used to test for a linear trend across time points.

\section{Power calculation}

The initial plan was to investigate the pre-treatment and post-treatment changes of $\mathrm{PGE}_{2}$ as an exploratory pilot study; thus no power calculations were performed at the planning stage. According to the values we observed in this study, the pre-treatment and post-treatment changes of $\mathrm{PGE}_{2}$ in $H$. pylori-positive (+) group were 16.8 (21) $\mathrm{pg} / \mathrm{mg}$ in the antrum and 27.5 (11) $\mathrm{pg} / \mathrm{mg}$ in the body. For a two-tailed 5\% type I error rate and $80 \%$ power, sample sizes of 244 and 28 for antrum and body, respectively, would be required to for these differences to reach statistical significance. Therefore, if the pretreatment and post-treatment changes in $\mathrm{PGE}_{2}$ can be attributed to COX-2 inhibition, our study does not have sufficient power to exclude the non-significant, likely non-clinically significant trend we observed.

\section{RESULTS}

$\mathrm{PGE}_{2}$ levels in gastric body and antrum

Before treatment, there was a significantly greater $\mathrm{PGE}_{2}$ level in the H. pylori (+) group compared with the H. pylori-negative (-) group for both the gastric body and antrum $(P<0.0001$ in both cases) (Figures 1 and 2$)$. In the body, the mean $\mathrm{PGE}_{2}$ was 111.5 (22.7) $\mathrm{pg} / \mathrm{mg}$ and $26.2(6.2) \mathrm{pg} / \mathrm{mg}$ for the H. pylori (+) and H. pylori $(-)$ subjects, respectively. In the antrum, the average $\mathrm{PGE}_{2}$ was 126.4 (25.7) $\mathrm{pg} / \mathrm{mg}$ and $35.2(4.9) \mathrm{pg} / \mathrm{mg}$ for the $H$. pylori $(+)$ and $H$. pylori (-) subjects, respectively.

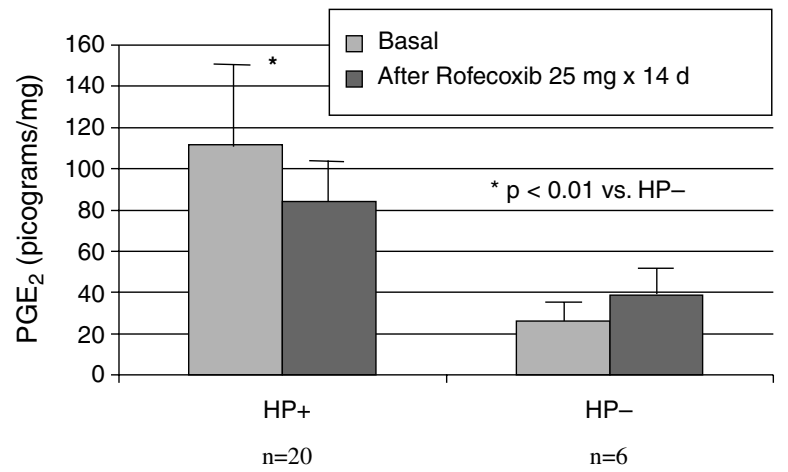

Figure 1. Concentrations of $\mathrm{PGE}_{2}$ in the biopsies of the gastric body from $H$. pylori-infected (HP,$+ n=20$ ) and uninfected (HP -, $n=6$ ) volunteers. The $\mathrm{PGE}_{2}$ levels were significantly higher in the infected stomachs than the control uninfected subjects.

Following treatment with rofecoxib $25 \mathrm{mg}$ for 14 days, there was a mean reduction of $25 \%$ in the HP + stomachs, which did not reach significance $(P>0.05)$. The $8 \%$ increase in the HP - group was also not significant.

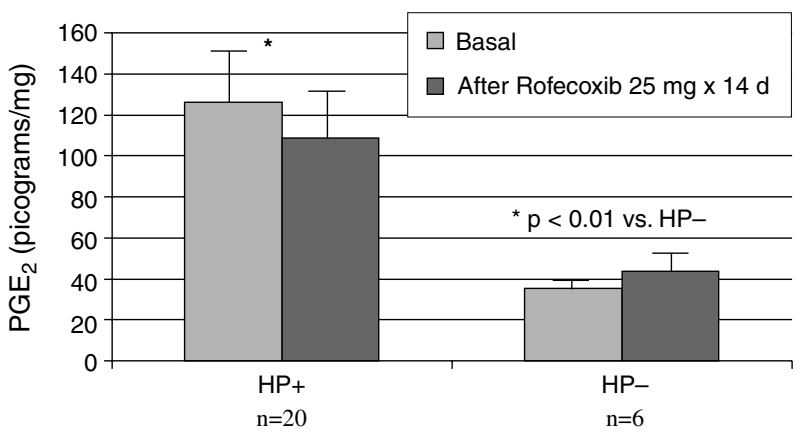

Figure 2. Concentrations of $\mathrm{PGE}_{2}$ in the biopsies of the antrum body from $H$. pylori-infected ( $\mathrm{HP}+, n=20)$ and uninfected (HP -, $n=6)$ volunteers. The $\mathrm{PGE}_{2}$ levels were significantly higher in the infected stomachs than the control uninfected subjects. Following treatment with rofecoxib $25 \mathrm{mg}$ for 14 days, there was a mean reduction of $13 \%$ in the HP + stomachs which did not reach significance $(P>0.05)$. The $11 \%$ increase in the HP group was also not significant.

Following 14 days of rofecoxib $25 \mathrm{mg}$ daily, subjects in the H. pylori (-) group showed non-significant 8 and $11 \%$ increases in $\mathrm{PGE}_{2}$ in the body and antrum. In the H. pylori (+) group there were non-significant decreases of $13 \%$ [95\% confidence interval (CI) $31 \%$ reduction to $17 \%$ increase] in the antrum and 25\% (95\% CI 55\% decrease to $3 \%$ increase) for $\mathrm{PGE}_{2}$ content. The gastric body average $\mathrm{PGE}_{2}$ levels increased to $12.3 \mathrm{pg} / \mathrm{mg}$ (13.9) in the H. pylori (-) group in contrast to an average decrease of 27.5 (11.1) pg/mg for the H. pylori $(+)$ group. In the antrum, there was an average 8.6 
(8.6) $\mathrm{pg} / \mathrm{mg}$ increase for the $H$. pylori (-) group in contrast to an average of $16.8(20.8) \mathrm{pg} / \mathrm{mg}$ decrease for the H. pylori (+) group. Examination of mean data supports a modest trend for the coxib to reduce $\mathrm{PGE}_{2}$ levels in the H. pylori-infected mucosa (Figures 1 and 2). However, examination of individual subject data did not demonstrate a consistent effect of inhibiting $\mathrm{PGE}_{2}$ in the H. pylori (+) subjects (Figure 3) The effect of rofecoxib on $\mathrm{PGE}_{2}$ levels was not significantly different in either group.

\section{Proliferation indices}

Before treatment, the proliferation index in middlegland mucosa was significantly higher in the H. pylori (+) group [antrum 58.6\% (2.3); body 48.5\% (3.3)] compared to the H. pylori (-) group [antrum 39.4\% (1.8); body 35.7\% (3.9)] (Figure 4). These differences were highly significant for both the gastric body and antrum $[P<0.001$ (antrum) and $P<0.005$ (body) .

After treatment there were no statistically significant changes in the proliferation indices in either the gastric
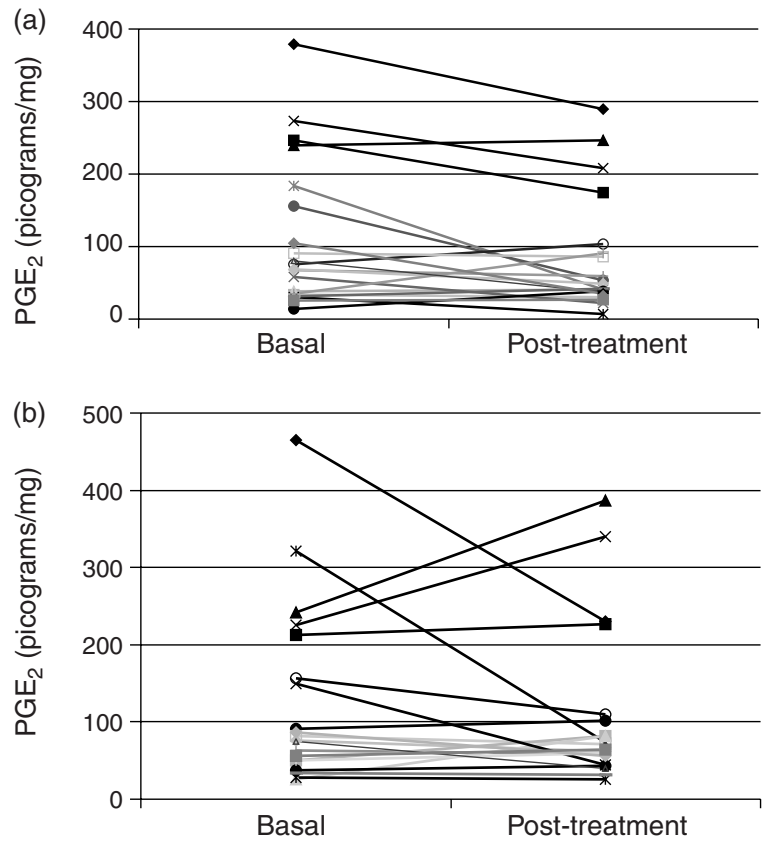

Figure 3. (a) Inidvidual $\mathrm{PGE}_{2}$ levels in the gastric body before and after rofecoxib $25 \mathrm{mg}$ for the $20 \mathrm{H}$. pylori-infected subjects. (b): Individual $\mathrm{PGE}_{2}$ levels in the gastric antrum before and after rofecoxib $25 \mathrm{mg}$ for the $20 \mathrm{H}$. pylori-infected subjects. The reduction in $\mathrm{PGE}_{2}$ is inconsitent, suggesting that $\mathrm{COX}-2$ is not the predominant source of increased $\mathrm{PGE}_{2}$ in the H. pylori-infected stomach.
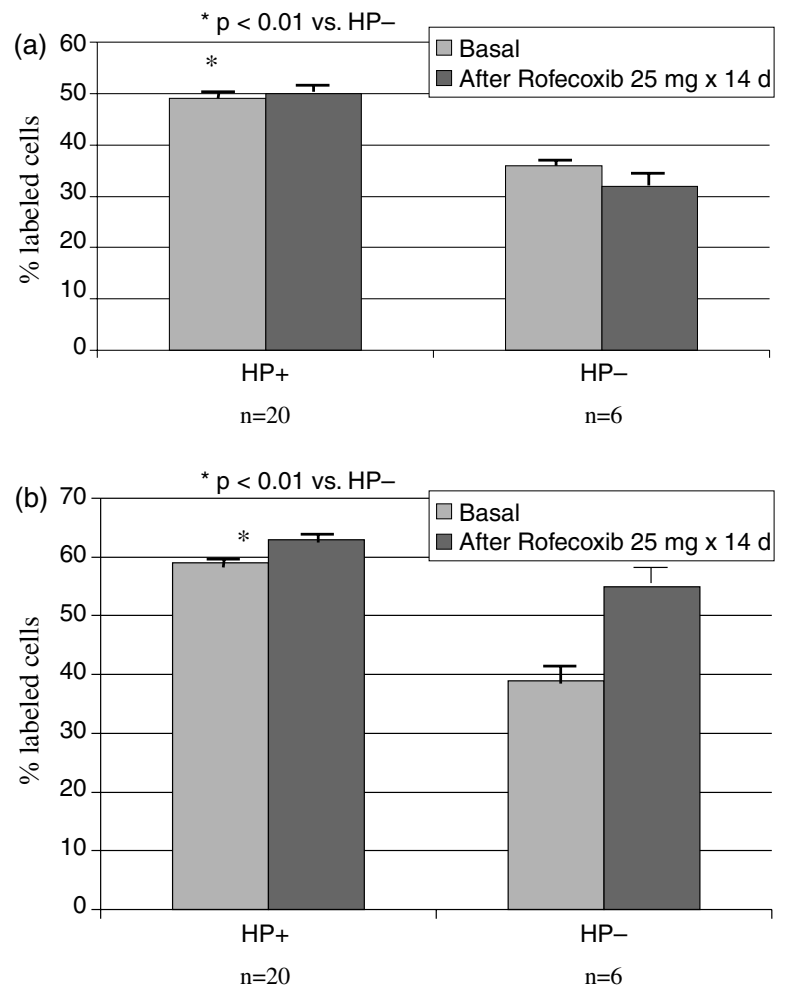

Figure 4. (a) Epithelial proliferation (\% of cells labelled with $\mathrm{Ki}-67)$ in the mid-zone of the gland in gastric body biopsies from H. pylori-infected ( $\mathrm{HP}+, n=20)$ and uninfected ( $\mathrm{HP}-, n=6)$ volunteers. (b) Epithelial proliferation in the mid-zone of the gland in gastric antral biopsies from H. pylori-infected ( $\mathrm{HP}+, n=20$ ) and uninfected (HP,$- n=6)$ volunteers. The proliferation levels were significantly higher in the infected stomachs than the control uninfected subjects. Following treatment with rofecoxib $25 \mathrm{mg}$ for 14 days, there was no change in proliferation in either group.

body or antrum. This result suggests that COX-2 inhibition had no significant effect on cell proliferation for H. pylori (+) or H. pylori (-) subjects.

\section{Gastritis severity}

Before treatment, the H. pylori (+) subjects had higher severity scores on all markers of gastritis. In the antrum, H. pylori density, polymorphonuclear neutrophil (PMN) scores and mononuclear scores were all significantly greater $(P<0.01)$ in the H. pylori $(+)$ group $[1.5(0.2)$, $1.1(0.2), 2(0.1)]$ compared to the H. pylori (-) group [0 $(0), 0(0), 0.5(0.2)]$. In the body, the degree of inflammation was less severe among the subjects, consistent with antral predominant infection. The H. pylori scores [0.9 (0.2) vs. $0(0)]$ and mononuclear 
scores [1.7 (0.1) vs. $0.5(0.3)]$ are significantly higher in the H. pylori $(+)$ groups $(P<0.05)$, but the PMN scores did not reach statistical significance $[1.7(0.2)$ vs. 0.5 (0.3), $P=0.29]$. The other components of the Sydney classification, atrophy and intestinal metaplasia, were too infrequently seen to analyse changes in response to drug. There were no significant differences in the effect treatment on gastritis severity for $H$. pylori (+) or H. pylori (-) subjects on either body or antrum. These results indicate that $\mathrm{COX}-2$ inhibition has no effect on the histological markers of gastritis severity.

\section{Endoscopy findings}

For those subjects free of $H$. pylori infection, following rofecoxib treatment for 14 days, no lesions developed in the stomach or antrum. For those with $H$. pylori infection, no lesions developed in the gastric body or duodenum. In the antrum, one subject developed two erosions, and a single subject developed an endoscopic ulcer.

\section{DISCUSSION}

The impact of the administration of a COX-2 specific inhibitors and non-selective NSAIDs on gastric prostaglandin levels has been studied in healthy human volunteers, but has not been previously characterized in the setting of H. pylori infection. In a previous study, when rofecoxib $50 \mathrm{mg}$ was given for 5 days to uninfected volunteers, a non-significant $18 \%$ increase in gastric mucosal $\mathrm{PGE}_{2}$ synthesis was observed, similar to our observations. ${ }^{11}$ In contrast, when naproxen, a dual COX inhibitor, was administered, gastric mucosa $\mathrm{PGE}_{2}$ synthesis was reduced by $65 \%$. As infection of human gastric mucosa by $H$. pylori is accompanied by mucosal inflammation and induction of COX-2 expression in both inflammatory cells and the epithelium, the impact of COX-2 inhibition in the setting of $H$. pylori infection is of keen scientific and clinical interest. ${ }^{16,17} \mathrm{H}$. pyloriassociated inflammation is accompanied by increases in gastric mucosal prostaglandin production, with $\mathrm{PGE}_{2}$ being the predominant prostaglandin end product evaluated in most studies. ${ }^{18}$

H. pylori infection is associated with two, largely divergent, important clinical outcomes for those individuals who carry the organism: peptic ulcer disease and gastric cancer. Indeed, the pathways for these clinical sequelae appear distinct, as patients who develop duodenal ulcer disease appear protected from cancer development, possibly due to differences in both infecting organism and host responses. ${ }^{19}$ The relationship between COX inhibition and H. pylori infection is relevant to both ulcer disease and cancer.

As the use of NSAIDs and infection with $H$. pylori are both very common, and each agent individually is an important peptic ulcerogen, great attention has been directed to these important causes of ulcer disease. To summarize a large and somewhat conflicting body of epidemiological and experimental data, the two agents appear to be independent risk factors for peptic ulcer disease. ${ }^{20}$ As NSAID-related ulcers are predominantly the result of COX-1 inhibition (or simultaneous COX-1 and COX-2 inhibition), COX-2 specific inhibitors were developed to avoid this risk associated with antiinflammatory drug therapy. However, the induction of COX-2 in the upper gastrointestinal tract in the setting of $H$. pylori infection raises the concern of increased ulcer risk with COX-2 inhibitors in $\mathrm{H}$. pylori-infected patients. While $H$. pylori-infected mucosa has been documented to have increased prostaglandin levels, the responsible COX isoform has not been identified by functional or inhibitor studies in humans. From a pathophysiological standpoint, this is not a minor concern, as the increase in prostaglandin levels in the infected stomach is substantial, and may play a role in limiting damage caused by the organism. ${ }^{18}$

Our data demonstrate that while the H. pylori-infected stomach has a higher mucosal $\mathrm{PGE}_{2}$ content compared to non-infected controls, the increase is largely attributable to COX-1-derived products. Although the size of our study limits the statistical power of our results, the data demonstrate that for the antrum and body of the stomach, reductions of $13-25 \%$ in the antrum and body, respectively, likely represent the greatest impact of COX-2-specific inhibition on the human gastric mucosa. However, the wide confidence intervals include no reduction to maximal reductions of 30-55\%. These data support the hypothesis that the increased prostaglandins in H. pylori-infected gastric mucosa are predominantly derived from $\mathrm{COX}-1$, and we hypothesize that no more than one third of the increased prostaglandin content observed in the $H$. pylori infected mucosa is derived from COX-2, although larger studies are necessary to accurately define this estimate. Our observations are consistent with previous studies that incubated H. pylori-infected mucosa derived from humans with specific COX-1 (sc58560) and COX-2 (N.S.-398) 
inhibitors ex vivo. ${ }^{21}$ Our data, using medications now widely utilized in clinical practice, both confirms and extends these clinically important observations.

Our data now provide support for the observations that, in the clinical trials of COX-2 inhibitors, H. pylori infection was not a risk factor for endoscopic or clinical ulceration. ${ }^{22}$ The endoscopic injury we observed in our study was mild, and was seen following rofecoxib in only in two H. pylori-infected subjects. Given the body of data supporting the independence of the effect of $H$. pylori on endoscopic injury on rofecoxib, it is tempting to speculate these simply may have been H. pylori-related lesions.

The recognition of $H$. pylori infection as precursor lesion for gastric epithelial and lymphoid malignancies has led to its classification as a class I carcinogen. The induction of COX-2 has been demonstrated to play an important role in gastrointestinal carcinogenesis for a number of tissues, particularly the colon and oesophagus. $^{23,}{ }^{24}$ As is the case for colorectal cancer, there is abundant epidemiological evidence that demonstrates that users of aspirin or traditional NSAIDs are at a reduced risk for gastric cancer. ${ }^{4}$ Also analogous to the colon, induction of COX-2 expression appears to play an important pathophysiological role in gastric carcinogenesis. $^{25}$ Thus, in concept, COX inhibition offers a potential thereapeutic strategy for chemoprevention in gastric cancer.

The risk of ulcers and bleeding precludes the use of non-selective NSAIDs as a chemopreventive strategy, particularly in the presence of another known ulcerogen such as $H$. pylori. While one approach, eradication of the infection, is a rational and potentially costeffective strategy to prevent gastric cancer, experimental evidence of the efficacy of this approach awaits long-term studies. ${ }^{26,27}$ The timing of such an intervention may be important, and the reversibility of changes such as intestinal metaplasia and atrophy remains unknown and potentially problematic. In addition, many high-risk populations for gastric cancer occur in environments with high reinfection rates, limiting the value of attempts at eradication. These issues are driving current studies aimed at vaccine development.

The lack of effect on intermediate biomarkers for neoplasia development following the administration of a specific COX-2 inhibitor raises uncertainty regarding their potential for chemoprevention of gastric cancer. The minimal impact of rofecoxib on prostaglandin levels was unassociated with changes in epithelial proliferation, in contrast to studies where non-selective COX inhibitors demonstrate profound inhibition of prostaglandin levels and associated reductions in the H. pylori-related increased mucosal proliferation. ${ }^{28,29}$ Our study cannot exclude the possibility that the mucosal response to COX-2-specific inhibition may vary among different subgroups of infected persons, as $H$. pylori strain differences may influence epithelial induction of $\mathrm{COX}-2,{ }^{25}$ and heterogeneity exists in the mucosal immune response. Given the observation that some of the $H$. pylori-infected subjects in this study appeared to respond with marked reductions in prostaglandin levels, further studies designed to characterize responders may prove valuable. Such additional studies suggested by our results may provide insight into the differential impact of infecting strain and the host response on the ultimate contributory role of the COX isoforms in $\mathrm{H}$. pylori gastritis.

Other potential explanations for our results include that there is actually a meaningful decrease in surrogate markers or neoplasia risk (e.g. prostaglandin proliferation) given the relatively small number of patients studied. Alternatively, COX inhibitors may lead to decreased neoplasia via other mechanisms that were not assessed, or gastric carcinogenesis may occur via mechanisms different from those in the colon and oesophagus, where COX-2 inhibitors have shown experimental evidence of an effect. As stated above, our study observations may be limited by the small sample size and the variation in $\mathrm{PGE}_{2}$ content among H. pylori (+) subjects. Furthermore, it is unclear how our observations might have changed had a nonselective NSAID been included in our study.

While our observations do raise the possibility that dual COX inhibition may be required for gastric chemoprevention; however, they do not preclude a potential chemopreventive role of COX-2-specific inhibitors in specific clinical situations. Analogous to Barrett's oesophagus, an area of intense interest for chemoprevention with COX-2 inhibitors, study of histological changes beyond the early changes of gastritis to tissues more highly programmed to cancer development, such as intestinal metaplasia or atrophic gastritis, should be the next area for investigation. ${ }^{30}$ These conditions are associated with epithelial hyperproliferation and increased COX-2 expression, and are ideal H. pylori-associated disease subgroups for the costeffective study of these agents in gastrointestinal chemoprevention. 
In summary, the administration of the COX-2-specific inhibitior rofecoxib, in healthy humans with $H$. pylori gastritis, failed to impact mucosal prostaglandin generation significantly or alter the observed H. pylorirelated mucosal hyperproliferation. These data support the following conclusions: (1) despite induction of COX-2 in the $H$. pylori-infected mucosa, the predominant source of increased prostaglandins in the H. pyloriinfected stomach is COX-1, (2) the lack of significant impact on these parameters supports the safety of COX-2 inhibitors in the H. pylori-infected upper gastrointestinal tract, and (3) the potential of COX-2 inhibitors for the chemoprevention of gastric cancer related to $H$. pylori infection remains uncertain. However, future studies in advanced preneoplastic conditions, such as atrophic gastritis and/or intestinal metaplasia, may be useful to explore this potential clinical application for these drugs.

\section{ACKNOWLEDGEMENTS}

This paper was presented in part as an oral presentation at the American Gastroenterology Association Meeting, May 2002. Grant support was received from the Comprehensive Cancer Center, University of Michigan and Merck, Inc.

\section{REFERENCES}

1 Scheiman JM, Cutler AF. Helicobacter pylori and gastric cancer. Am J Med 1999; 106: 222-6.

2 Ruiz B, Garay J, Correa P, et al. Morphometric evaluation of gastric antral atrophy: improvement after cure of Helicobacter pylori infection. Am J Gastroenterol 2001; 96: 3281-7.

3 Sung JJ, Lin SR, Ching JY, et al. Atrophy and intestinal metaplasia one year after cure of $H$. pylori infection: a prospective, randomized study. Gastroenterology 2000; 119: 7-14.

4 Krishnan K, Brenner DE. Prostaglandin inhibitors and the chemoprevention of noncolonic malignancy. Gastroenterol Clinics North Am 2001; 30: 981-1000.

5 Sung JJ, Leung WK, Go MY, et al. Cyclooxygenase-2 expression in Helicobacter pylori-associated premalignant and malignant gastric lesions. Am J Pathol 2000; 157: 729-35.

6 Ristimaki A, Honkanen N, Jankala H, et al. Expression of cyclooxygenase-2 in human gastric carcinoma. Cancer Res 1997; 57: 1276-80.

7 Zhu GH, Yang XL, Lai KC, et al. Nonsteroidal antiinflammatory drugs could reverse Helicobacter pylori-induced apoptosis and proliferation in gastric epithelial cells. Dig Dis Sci 1998; 43: 1957-63.

8 Hudson N, Balsitis M, Filipowicz F, et al. Effect of Helicobacter pylori colonisation on gastric mucosal eicosanoid synthesis in patients taking non-steroidal anti-inflammatory drugs. Gut 1993; 34: 748-51.

9 Hawkey C, Laine L, Simon TE, et al. Comparison of the effect of rofecoxib (a cyclooxygenase 2 inhibitor), ibuprofen, and placebo on the gastroduodenal mucosa of patients with osteoarthritis. Arth Rheum 2000; 43: 370-7.

10 Bombardier C, Laine L, Reicin A, et al. Comparison of upper gastrointestinal toxicity of rofecoxib and naproxen in patients with rheumatoid arthritis. N Engl J Med 2000; 343: 1505-84.

11 Wright NJ, Gottesdiener K, Garlick NM, et al. Rofecoxib, a COX-2 inhibitor, does not inhibit human gastric mucosal prostaglandin production. Gastroenterology 2001; 120: 867-73.

12 Hawkey CJ, Laine L, Harper SE, et al. Influence of risk factors on endoscopic and clinical ulcers in patients taking rofecoxib or ibuprofen in two randomized controlled trials. Aliment Pharmacol Ther 2001; 15: 1593-601.

13 Chan FK, To KF, Ng YP, et al. Expression and cellular localization of COX-1 and -2 in Helicobacter pylori gastritis. Aliment Pharmacol Ther 2001; 15: 187-93.

14 Cryer B, Feldman M. Effects of very low dose daily, long-term aspirin therapy on gastric, duodenal, and rectal prostaglandins levels and on mucosal injury in healthy humans. Gastroenterology 1999; 117: 7-25.

15 Dixon MF, Genta RM, Yardley JH, et al. Classification and grading of gastritis: The updated Sydney System. Am J Surg Pathol 1996; 20: 1161-81.

16 McCarthy CJ, Crofford LJ, Greenson J, et al. Cyclooxygenase-2 expression in gastric antral mucosa before and after eradication of Helicobacter pylori infection. Am J Gastroenterol 1999; 94: 1218-23.

17 Fu S, Ramanujam KS, Wong A, et al. Increased expression and cellular localization of inducible nitric oxide synthase and cyclooxygenase 2 in Helicobacter pylori gastritis. Gastroenterology 1999; 116: 1319-29.

18 Feldman M, Cryer B, Mallat D, Go MF. Role of Helicobacter pylori infection in gastroduodenal injury and gastric prostaglandin synthesis during long term/low dose aspirin therapy: a prospective placebo-controlled, double-blind randomized trial. Am J Gastroenterol 2001; 96: 1751-7.

19 Hansson LE, Nyren O, Hsing AW, et al. The risk of stomach cancer in patients with gastric cancer or duodenal ulcer disease. N Engl J Med 1996; 335: 242-9.

20 Huang JQ, Sridhar S, Hunt RH. Role of Helicobacter pylori infection and non-steroidal anti-inflammatory drugs in pepticulcer disease: a meta-analysis. Lancet; 2002; 359(9300): 14-22.

21 Jackson LM, Wu KC, Mahida YR, et al. Cyclooxygenase (COX) 1 and 2 in normal inflamed and ulcerated human gastric mucosa. Gut 2000; 47: 762-70.

22 Laine L, Bombardier C, Hawkey C, et al. Stratifying the risk of NSAID-related upper gastrointestinal clinical events: results of a double-blind outcomes study in patients with rheumatoid arthritis. Gastroenteol 2002; 123: 1006-12.

23 Gupta RA, Dubois RN. Colorectal, cancer prevention and treatment by inhibition of cyloxygenase-2. Natl Rev Cancer 2001; 1: 11-21. 
24 Hussain SS, Szabo IL, Tarnawski AS. NSAID Inhibition of GI cancer growth: clinical implications and molecular mechanisms of action. Am J Gastoenterol 2002; 97: 542-53.

25 Romano M, Ricci V, Memoli A, et al. Helicobacter pylori up-regulates cyclooxygenase-2 mRNA expression and prostaglandin $\mathrm{E}_{2}$ synthesis in MKN 28 gastric mucosal cells in vitro. J Bio Chem 1998; 273: 28 560-3.

26 Fendrick AM, Chernew ME, Hirth RA, et al. Clinical and economic effects of $H$. pylori screening to prevent gastric cancer. Arch Int Med 1999; 159: 142-8.

27 Fraser AG, Sim R, Sankey EA, et al. Effect of eradication of Helicobacter pylori on gastric epithelial cell proliferation. Aliment Pharm Ther 1994; 8: 167-73.
28 Levi S, Goodlad RA, Lee CY, et al. Effects of nonsteroidal antiinflammatory drugs and misoprostol on gastroduodenal epithelial proliferation in arthritis. Gastroenterology 1992; 102: 1605-11.

29 Tseng GY, Lin HJ, Lin HY, et al. Effect of non-steroidal antiinflammatory drugs on gastric and duodenal prostaglandin concentrations in patients with Helicobacter pylori infection. Hepatogastroenterology 1999; 46: 1000-4.

30 Kaur BS, Khamnehei N, Iravani M, et al. Rofecoxib inhibits cyclooxygenase 2 expression and activity and reduces cell proliferation in Barrett's esophagus. Gastroenterology 2002; 123: $60-7$. 